\title{
Necrosectomía endoscópica asistida por laparoscopia: un enfoque efectivo en la necrosis pancreática infectada
}

\author{
Flávia Fernanda de Françal, Izabella Maria Lopes Titon ${ }^{1}$, \\ Jonas Takada', Nickson Santana Souto ${ }^{3}$, Rafael Antunes Delfes ${ }^{4}$, \\ Fernanda Kreve y Francisco Schossler Loss ${ }^{5}$
}

\section{Endoscopic necrosectomy assisted by laparoscopy: an effective approach in infected pancreatic necrosis}

Acute pancreatitis is a disease characterized by intense inflammation caused by the degradation of the parenchyma and adjacent tissue, through activation and consequent protease output. Approximately $15 \%$ of patients with the disease progress to necrotizing form and subsequent infection of this necrotic tissue is a complex process and associated with significant morbidity and mortality. Interventional treatment is mandatory in the management of infected necrosis and open surgery has perpetuated for decades as a first-line treatment to remove infected necrotic tissue. In recent years, with the improvement in laparoscopy and other techniques, new approaches for the management of this condition have been advocated to the detriment of open surgery, in particular, the advent of the Step-up approach. This new approach allowed the management of infected necrosis in a gradual way. Percutaneous drainage is the first step, and is effective by itself in up to $35 \%$ of cases, then, if there is no clinical remission or if the collection drainage is not satisfactory, the invasiveness of the process increases. Techniques such as video-assisted retroperitoneal debridement (VARD) and endoscopic transgastric necrosectomy (ETN) are examples of minimally invasive procedures widely used and applied in these cases, as they allow access to the necrotic collection with minimal aggression, in addition to inducing low physiological stress, resulting in a lower incidence of unfavorable outcomes. We describe a case of a patient, male, 64 years old, hypertensive, with infected necrotizing pancreatitis, with 6 weeks of evolution. Following the parameters of the Step-up approach, treatment started with percutaneous drainage to resolve the acute condition and, subsequently, an alternative technique was used, employing endoscopic necrosectomy assisted by laparoscopy. The management of this case demonstrates an effective and fully feasible technique, which can be replicated in other hospital centers.

Key words: Video-assisted retroperitoneal debridement (VARD), Endoscopic transgastric necrosectomy (ETN), Step-up approach, Minimally invasive, Walled-off pancreatic necrosis.

\section{Resumen}

La pancreatitis aguda es una enfermedad caracterizada por una intensa inflamación del páncreas. Aproximadamente el $15 \%$ de los pacientes con la enfermedad progresan a una forma necrosante y la infección posterior de este tejido necrotizado es un proceso complejo y asociado con una morbilidad y mortalidad significativas. El tratamiento intervencionista es obligatorio, en la mayoría de los casos, en el manejo de la necrosis infectada y la cirugía abierta se ha perpetuado durante décadas como tratamiento de primera línea para eliminar el tejido necrótico infectado. En los últimos años, con la mejora de la laparoscopia y otras técnicas, se han defendido nuevos enfoques para el manejo de esta afección en detrimento de la cirugía abierta, en particular, el advenimiento del enfoque Step-up. Este nuevo enfoque permitió el manejo de la necrosis infectada de forma gradual. El drenaje percutáneo es el primer paso, y es efectivo por sí solo hasta en el 35\% de los casos, luego, si no hay remisión clínica o si el drenaje de la colección no es satisfactorio, aumenta la invasividad del proceso. Técnicas como el desbridamiento retroperitoneal videoasistido (VARD) y la necrosectomía transgástrica endoscópica (ETN) son ejemplos de procedimientos mínimamente invasivos ampliamente utilizados y aplicados en estos casos, ya que permiten acceder a la colección necrótica con mínima agresión, además de inducir poco estrés
'Estudiante de

medicina. Universidad Federal de Paraná.

Toledo, Brasil.

${ }^{2}$ Departamento de

Cirugía, Universidad de São Paulo. Toledo, Brasil.

${ }^{3}$ Médico. Universidad Estatal de Montes

Claros. Montes Claros,

Brasil.

${ }^{4}$ Radiología y

diagnóstico por

imagen, Hospital

Universitário

Evangélico de

Curitiba. Curitiba,

Brasil.

${ }^{5}$ Académico de la

Facultad de Medicina,

Centro Universitário

Fundação Assis

Gurgacz. Cascavel,

Brasil.

Recibido: 18 de agosto de 2020

Aprobado: $1 \mathrm{de}$ noviembre de 2020

Correspondencia a: Dr. Francisco Schossler Loss

Calle Souza Naves

4013 Cascavel / PR

Brasil.

Código postal

85810070 .

franciscoloss@

hotmail.com 
fisiológico, resultando una menor incidencia de resultados desfavorables. Describimos el caso de un paciente, varón, 64 años, hipertenso, con necrosis pancreática infectada, con 6 semanas de evolución. Siguiendo los parámetros del abordaje Step-up, el tratamiento se inició con drenaje percutáneo para la resolución del cuadro agudo y, posteriormente, se utilizó una técnica alternativa, empleando una necrosectomía endoscópica asistida por laparoscopia. El manejo de este caso demuestra una técnica eficaz y plenamente factible, que puede replicarse en otros centros hospitalarios.

Palabras clave: Desbridamiento retroperitoneal videoasistido (VARD), Necrosectomía endoscópica transgástrica (ETN), Abordaje escalonado, Mínimamente invasivo, Necrosis pancreática de límites definidos.

\section{Introducción}

La pancreatitis aguda es una enfermedad caracterizada por una intensa inflamación del páncreas, que puede conducir a cambios estructurales locales y sistémicos, e incluso puede culminar en desenlaces fatales ${ }^{1}$. La forma necrosante de la enfermedad se informa en $15 \%$ de los pacientes y se define como necrosis del parénquima o tejido peripancreático ${ }^{2}$. El resultado negativo más relevante en la evolución de esta condición es la infección, que promueve un aumento de la mortalidad de 12 al $39 \% \%^{2,3}$.

En pacientes con necrosis no infectada, se puede aplicar un tratamiento conservador. En la mayoría de los casos, la necrosis pancreática infectada debe abordarse mediante técnicas de intervención ${ }^{4}$. Tradicionalmente, el abordaje del tejido necrótico e infectado se realizaba mediante laparotomía estándar ${ }^{4}$. Sin embargo, debido a las altas tasas de complicaciones (34 a 95\%) y mortalidad (11 a 39\%), su manejo ha sufrido importantes cambios de paradigma ${ }^{5,6}$.

Actualmente, los abordajes mínimamente invasivos (realizados de forma aislada o secuencial) como el drenaje percutáneo (DP), la necrosectomía endoscópica transgástrica (ETN) y el desbridamiento retroperitoneal videoasistido (VARD), se han convertido en opciones terapéuticas de elección, ya que han reducido la tasas de mortalidad y complicaciones en una enfermedad compleja $\mathrm{j}^{4,7,8}$.

Describimos el caso de un paciente con necrosis pancreática infectada de 6 semanas de evolución, tratado de forma sencilla, segura y eficaz mediante técnica de necrosectomía endoscópica asistida por laparoscopia.

\section{Reporte de caso}

Paciente masculino, 64 años, hipertenso. Acude a atención médica tras ingreso hospitalario por pancreatitis aguda complicada, con una evolución de 6 semanas. Tenía dolor en la región abdominal superior, asociado a hiporexia y fiebre (38 grados). Las pruebas de laboratorio mostraron leucocitos de 14.600, amilasa sérica $36 \mathrm{U} / \mathrm{L}$, lipasa $50 \mathrm{U} / \mathrm{L}$, TGO $54 \mathrm{U} / \mathrm{L}$, TGP 42 U/L y PCR $80,9 \mathrm{mg} / \mathrm{L}$ y hemocultivo negativo.

Se solicitó tomografía computarizada de abdomen con contraste intravenoso (Figura 1), que mostró áreas de necrosis bien definidas adyacentes a cabeza, cuerpo y cola pancreática, con presencia de burbujas de aire en su interior, compatible con el diagnóstico de necrosis pancreática infectada de límites definidos. Durante el procedimiento se administró antibiótico (ciprofloxacino y metronidazol) y se mantuvieron durante todo el seguimiento durante 3 semanas.

Ante los hallazgos, optamos por el drenaje percutáneo guiado por tomografía a través de un catéter en "pig tail" de 16 french, que por su proximidad a la pared lateral del flanco anterior izquierdo se insertó en una topografía anterior al mesocolon del colon izquierdo (Figura 2).

El paciente tuvo una mejoría significativa de su estado infeccioso, pero al quinto día de seguimiento mantuvo dolor abdominal persistente y supuración purulenta. La tomografía computarizada de control mostró poca regresión del compartimento del área de necrosis pancreática (Figura 3).

Debido a la evolución y persistencia no del todo satisfactoria de la cavidad de necrosis pancreática, optamos por realizar la necrosectomía de la cavidad establecida de forma mínimamente invasiva, utilizando como guía el catéter de drenaje percutáneo.

Bajo anestesia general, se colocó al paciente en decúbito supino con la mesa de operaciones lateralizada 30 grados hacia el lado derecho. El brazo izquierdo se colocó cerca del cuerpo del paciente. Inicialmente, se realizaron tres incisiones en las regiones del ombligo (para la inserción de la cámara de laparoscopia), hipocondrio derecho y flanco izquierdo (línea media izquierda, a la altura del ombligo) para la inserción de 2 trócares de $5 \mathrm{~mm}$ después de la creación del neumoperitoneo.

La vista laparoscópica permitió visualizar intraabdominalmente el catéter en "pig tail" previamente ubicado en el almacén de necrosis, emergiendo de la pared abdominal anterolateral y perforando el 


\section{Caso clínico}

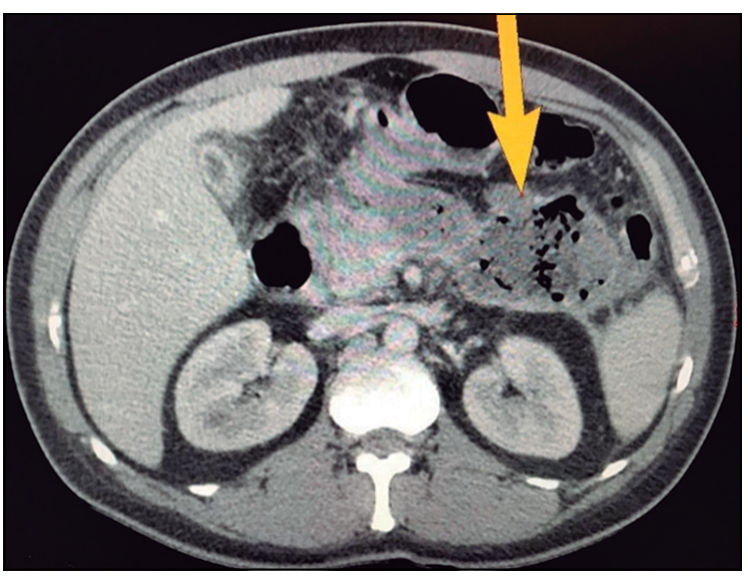

Figura 1. Tomografía computarizada de abdomen que muestra un área infectada de necrosis pancreática de límites definidos.

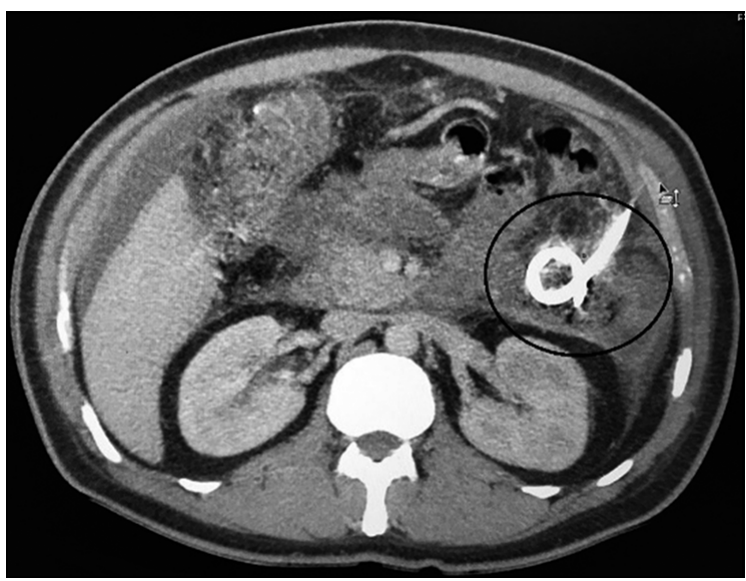

Figura 3. Tomografía computarizada de control (quinto día) que muestra una regresión discreta del compartimento del área de necrosis pancreática, incluso con una buena colocación del drenaje percutáneo.

mesocolon del colon transverso. Paralelamente a este catéter, insertamos un trócar de válvula de plástico desechable de $12 \mathrm{~mm}$ en el depósito pancreático infectado de forma sencilla. Este trocar permitió realizar el desbridamiento directo con un video endoscópico flexible de $9,8 \mathrm{~mm}$, con excelente visualización de la cavidad de necrosis (Figura 4).

Se extrajeron porciones más grandes de tejido necrótico con la ayuda de pinzas de basket y se limpió la cavidad con solución salina caliente. La técnica utilizada para el desbridamiento utilizó solo materiales básicos de endoscopia y laparoscopia. El procedimiento se realizó en un tiempo total de $90 \mathrm{~min}$ (Figura 5).

Se retiró el drenaje percutáneo y se insertó una

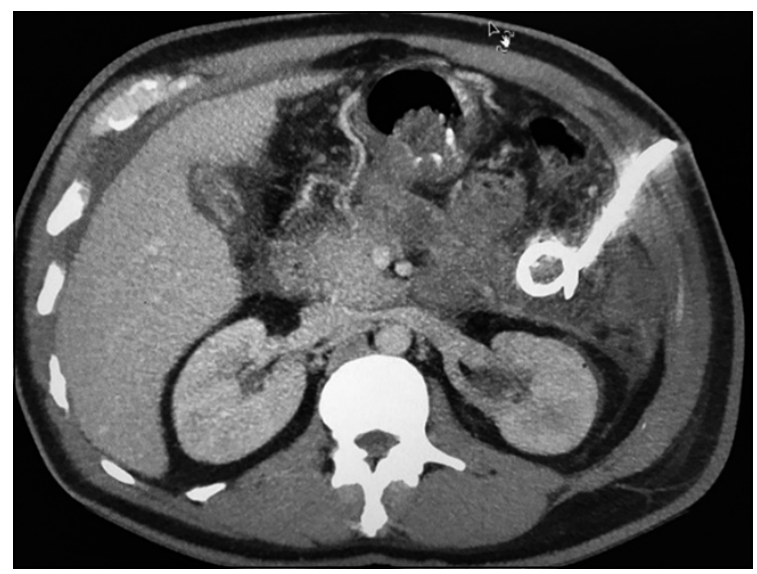

Figura 2. Tomografía computarizada que muestra un catéter de drenaje percutáneo ubicado en la cavidad de la necrosis pancreática infectada.

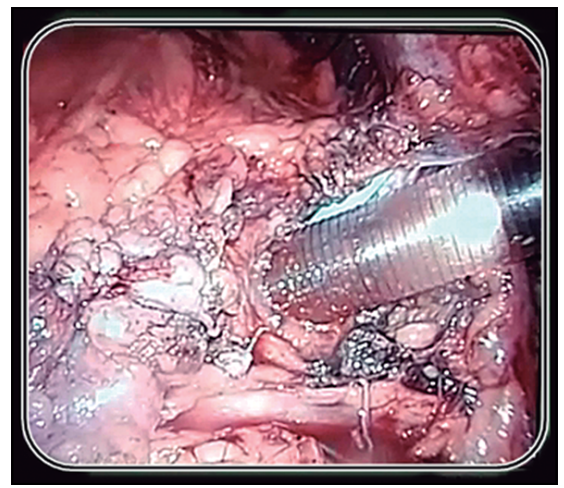

Figura 4. Vista laparoscópica del trocar insertado en la cavidad de necrosis, paralelo al catéter de drenaje percutáneo previamente ubicado.

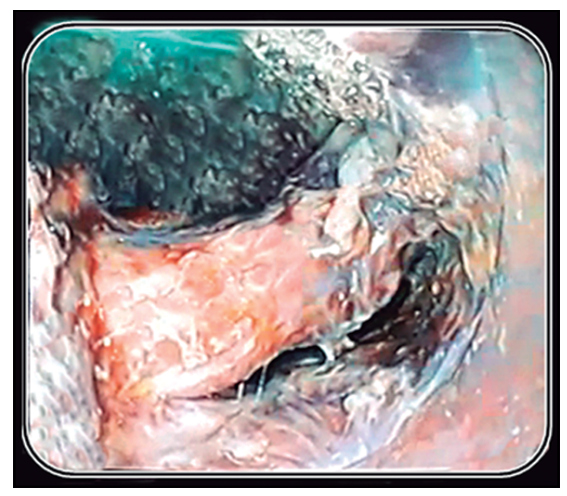

Figura 5. Vista endoscópica de la cavidad establecida por necrosis pancreática.

sonda vesical de Foley calibrada de 3 vías en la cavidad pancreática a través de la incisión utilizada para el paso del trócar en el flanco izquierdo. Con el manguito inflado en la cavidad necrótica desbridada, fue posible realizar infusiones posteriores con solución salina y al mismo tiempo monitorear el flujo y apariencia de las secreciones drenadas después del 


\section{Caso clínico}

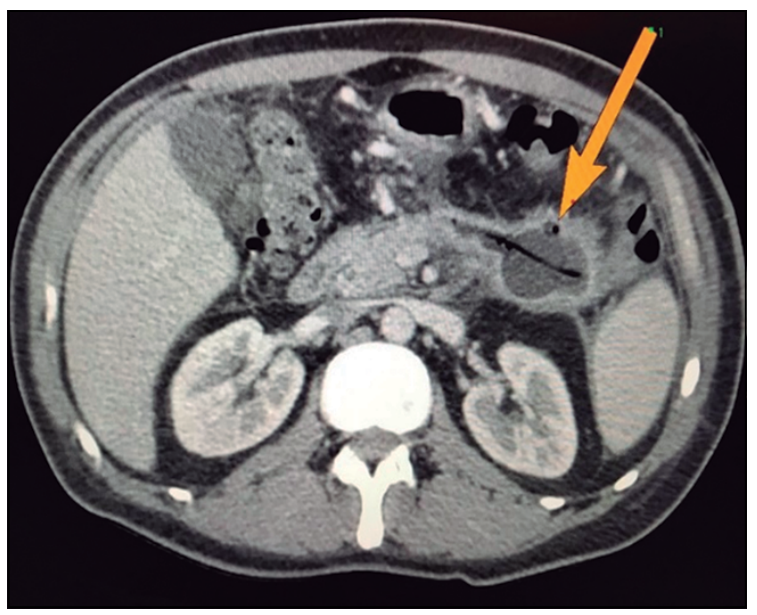

Figura 6. Tomografía computarizada que muestra una disminución significativa en la cavidad necrótica después de 3 semanas de necrosectomía asistida por video endoscópica. La flecha apunta al manguito de la sonda de Foley inflado en la cavidad necrótica.

procedimiento. La sonda se suturó a la piel y se rodeó por una bolsa de estoma.

La dieta se introdujo el primer día postoperatorio y la paciente evolucionó sin complicaciones, siendo dada de alta al tercer día. En una semana de seguimiento, las pruebas de laboratorio volvieron a establecer parámetros normales.

En la tercera semana postoperatoria, observamos una reducción significativa de la cavidad necrótica y las colecciones por tomografía computarizada (Figura 6). Retiramos la sonda vesical en 2 meses por prudencia y no por evolución desfavorable. Al final de la tercera semana postoperatoria, se suspendió la terapia con antibióticos. El flujo diario de la sonda promedió $5 \mathrm{ml}$ por día, que regresó a flujo cero en las siguientes semanas. Luego de completar 2 meses de postoperatorio, la sonda fue retirada por prudencia, considerando la deuda nula sostenida como uno de los criterios para su retirada. Se realizaron tomografías computarizadas de control tardías después de retirar la sonda, lo que confirmó la regresión completa de la cavidad necrótica.

\section{Discusión}

La clasificación de Atlanta (revisada en 2012), define que después de 4 semanas de pancreatitis aguda pueden ocurrir acumulaciones con formación de pseudoquistes pancreáticos -que tienen secreción de líquido homogénea- y necrosis pancreática delimitada (NPD), esto ocurre en hasta $10 \%$ de casos que se asocian a colección líquida heterogénea con restos necróticos del páncreas ${ }^{9-12}$.
En 40 al 70\% de los pacientes con NPD se instala una importante infección bacteriana secundaria, con un marcado factor de riesgo de mortalidad tardía. Este escenario se acompaña de deterioro clínico y de laboratorio, requiriendo antibióticos y tratamiento invasivo que incluye drenaje y necrosectomía ${ }^{13}$.

En estas situaciones, la laparotomía para necrosectomía pancreática ha sido considerada durante mucho tiempo para el tratamiento quirúrgico de elección, incluso si abre el tejido infectado y contiene la infección ${ }^{5}$. Sin embargo, debido a la alta tasa de mortalidad (11 a 39\%) y al riesgo de diseminación de la infección, la cirugía abierta como primera intervención se considera obsoleta y contraindicada desde las últimas 2 décadas $^{5}$.

En vista de este escenario, las terapias mínimamente invasivas, incluido el drenaje percutáneo o endoscópico, asociadas con una necrosectomía menos invasiva, han reemplazado radicalmente el manejo de la pancreatitis necrotizante infectada ${ }^{2}$. Dichas técnicas empleadas de forma gradual (Step-up approach) se basan en el control del foco infeccioso y el posterior desbridamiento del tejido necrótico según sea necesario $^{14}$.

El primer paso del "Step-up approach" consiste en el drenaje (radiológico percutáneo o endoscópico intraluminal) según la ubicación y proximidad o no a órganos adyacentes del compartimento infectado $0^{8,15,16}$. La justificación del procedimiento es controlar el foco infeccioso, mejorando el estado clínico del paciente, permitiendo incluso la resolución completa de la enfermedad hasta en $35 \%$ de los casos $^{16}$.

Cuando no se espera una mejoría clínica o se considera que las colecciones no se drenaron lo suficiente, es necesario un desbridamiento mínimamente invasivo como un segundo paso del proceso ${ }^{14}$. Para minimizar el estrés quirúrgico, dos técnicas ampliamente utilizadas son la necrosectomía transluminal endoscópica (ETN) y el desbridamiento retroperitoneal videoasistido (VARD) $)^{6,10}$.

El desbridamiento endoscópico se realiza tras un tracto digestivo fistuloso (gástrico o duodenal) con la colección necrótica pancreática delimitada en el retroperitoneo ${ }^{17}$. Predeterminado o no con la ayuda de la endosonografía, el camino debe ampliarse con la ayuda de dilatadores de balón especializados. Asimismo, debido a la posible necesidad de múltiples sesiones de desbridamiento endoscópico, el recorrido se mantiene a través del paso de prótesis autoexpandibles plásticas o metálicas ${ }^{18}$. El procedimiento endoscópico ciertamente evita la laparotomía, con su alta respuesta inflamatoria y sus complicaciones, pero combina costes y se asocia a complicaciones que no suelen ser graves, como hemorragia (9\%), fístulas, oclusión o migración de prótesis ${ }^{11,17,19,20}$.

Otra opción es la técnica VARD, que consiste en 
realizar una incisión de $5 \mathrm{~cm}$ en el flanco izquierdo utilizando el camino definido por el drenaje percutáneo para acceder a la necrosis pancreática ${ }^{2,7}$. Se inserta una óptica rígida de laparoscopia y se realiza necrosectomía con el uso de instrumentos utilizados en cirugías convencionales ${ }^{2}$. La rigidez de la óptica y los instrumentos dificulta la ejecución de este enfoque en las cavidades a menudo complejas determinadas por la necrosis y el proceso inflamatorio inherentes a la pancreatitis aguda. Además, se requiere un promedio de 3 reintervenciones y ocurren complicaciones en $35 \%$ de los abordajes, entre ellas hemorragia (17\%), fístulas entéricas $(9 \%)$, perforación de las vísceras $(4 \%)$ y hernia incisional $(3 \%)^{7}$.

Según la literatura, también se describen otras técnicas, como la técnica de acceso percutáneo a la necrosis pancreática con un stent autoexpandible. En esta técnica, se inserta un stent autoexpandible cubierto en un túnel fistuloso creado previamente para su expansión. Posteriormente se realizan varias sesiones de inserciones endoscópicas y extracciones necróticas. Es de destacar que la presente técnica tiene un alto costo para su realización, muchas veces con poca adherencia por parte de los servicios hospitalarios. Además, la trayectoria proporcionada puede no mantenerse debido a la inestabilidad del stent. Además, la creación de una cavidad ideal puede verse obstaculizada por el hecho de que el stent no está valvulado ${ }^{21}$.

El beneficio del Step-up approach, que evita la cirugía mayor, reduce no solo la tasa de complicaciones tempranas sino también la disfunción multiorgánica, sino que también disminuye la posibilidad de diabetes y hernias incisionales a largo plazo ${ }^{18}$. Tras el drenaje inicial percutáneo o endoscópico, la elección de un abordaje adicional videoasistido endoscópico o retroperitoneal depende de la ubicación de la cavidad determinada por la pancreatitis, así como de la expe- riencia y disponibilidad de las tecnologías presentes en un determinado servicio ${ }^{8,18}$.

La presentación tardía del cuadro (6 semanas) en nuestro paciente permitió tomar la decisión de iniciar el procedimiento invasivo sobre un tejido necrótico infectado bien diseñado. La colocación del drenaje percutáneo anterior al mesocolon del colon izquierdo (y no en la posición retroperitoneal) no solo mejoró la condición clínica del paciente, sino que ciertamente facilitó la visualización del drenaje en la cavidad durante la laparoscopia, reduciendo los riesgos de posibles lesiones en los órganos adyacentes.

Por su ubicación cercana a la pared abdominal y con el objetivo de adecuar la situación a los costes, el desbridamiento retroperitoneal videoasistido fue una opción. Sin embargo, considerando la complejidad e irregularidad de la lesión, la inserción de un endoscopio flexible permitió el uso de instrumentos adecuados y maniobras dentro de la cavidad que son fáciles de realizar. En nuestra opinión, esto no solo permitió un enfoque de una sola vez, sino que también disminuyó la posibilidad de lesiones inadvertidas. Además, también redujo considerablemente los costos, ya que no se utilizaron materiales complejos, no hubo complicaciones y no se requirieron nuevas intervenciones.

Se concluye que la idea de combinar 2 procedimientos (laparoscopia y endoscopia) permitió maximizar el manejo de una necrosis pancreática compleja infectada, con poco riesgo para el paciente, haciendo que el método sea seguro, exitoso y efectivo. Además, consideramos que el procedimiento es factible y replicable en la mayoría de unidades hospitalarias, ya que los materiales empleados en el abordaje ya son de uso rutinario por varios cirujanos. Tanto desde el punto de vista clínico como económico, consideramos que la técnica aporta importantes ventajas en un abordaje mínimamente invasivo.

\section{Referencias}

1.- Cunha EF de C, Rocha M de S, Pereira FP, Blasbalg R, Baroni RH. Necrose pancreática delimitada e outros conceitos atuais na avaliação radiológica da pancreatite aguda. Radiol Bras. 2014;47:165-75.

2.- Rasslan R, Novo F da CF, Bitran A, Utiyama EM, Rasslan S. Management of infected pancreatic necrosis: state of the art. Rev. Col. Bras. Cir. 2017;44:521-9.

3.- Werge M, Novovic S, Schmidt PN, Gluud LL. Infection increases mortality in necrotizing pancreatitis: A systematic review saand meta-analysis

Pancreatology 2016;16:698-707.

4.- Hackert T, Büchler MW. Decision

Making in Necrotizing Pancreatitis. Dig Dis 2016;34:517-24

5.- Raraty MG, Halloran CM, Dodd

S, Ghaneh P, Connor S, Evans J, et al. Minimal Access Retroperitoneal Pancreatic Necrosectomy: Improvement in Morbidity and Mortality With a Less Invasive Approach. Ann Surg. 2010;251:787-93.

6.- Van Brunschot S, Besselink MG, Bakker
OJ, Boermeester MA, Gooszen HG, Horvath KD, van Santvoort HC. VideoAssisted Retroperitoneal Debridement (VARD) of Infected Necrotizing Pancreatitis: An Update. Curr Surg Rep 2013;1:121-30

7.- Logue JA, Carter CR. Minimally Invasive Necrosectomy Techniques in Severe Acute Pancreatitis: Role of Percutaneous Necrosectomy and Video-Assisted Retroperitoneal Debridement. Gastroenterol Res Pract. 2015;2015:693040. 


\section{Caso clínico}

8.- Sorrentino L, Chiara O, Mutignani M, Sammartano F, Brioschi P, Cimbanassi S. Combined totally mini-invasive approach in necrotizing pancreatitis: a case report and systematic literature review. World J Emerg Surg. 2017;12:16.

9.- Banks PA, Bollen TL, Dervenis C, Gooszen HG, Johnson CD, Sarr $\mathrm{MG}$, et al. Classification of acute pancreatitis-2012: revision of the Atlanta classification and definitions by international consensus. Gut 2012;62:102-11.

10.- Moura CL, Barros P, Oliveira CM, Colaiácovo R, Drigo JM, Carbonari A, Campos T, Rossini L. Minimally invasive endoscopic treatment of necrotizing pancreatitis: A case report with images and review of the literature. Rev Assoc Med Bras 2017;63:207-9.

11.- Gardner TB. Endoscopic management of necrotizing pancreatitis. Gastrointest Endosc 2012;76;1214-23.

12.- Voermans RP, Fockens P. Endoscopic transluminal drainage of walled-off necrosis: Does size matter? Gastrointest Endosc 2016; 83: 708-10.
13.- Mazaki T, Ishii Y, Takayama T. Metaanalysis of prophylactic antibiotic use in acute necrotizing pancreatitis. $\mathrm{Br} \mathrm{J}$ Surg 2006;93:674-84.

14.- Sion MK, Davis KA. Step-up approach for the management of pancreatic necrosis: a review of the literature. Trauma Surg Acute Care Open 2019;4:e000308.

15.- Besselink MGH. The "Step-up approach" to infected necrotizing pancreatitis: Delay, drain, debride. Dig Liver Dis. 2011;43:421-2

16.- van Santvoort HC, Besselink MG, Bakker OJ, Hofker HS, Boermeester MA, Dejong CH, et al. A Step-up Approach or Open Necrosectomy for Necrotizing Pancreatitis. N Engl J Med 2010;362:1491-502.

17.- Van Brunschot S, Fockens P, Bakker OJ, Besselink MG, Voermans RP, Poley JW, et al. Endoscopic transluminal necrosectomy in necrotising pancreatitis: a systematic review. Surg Endosc 2014; 28, 1425-38.

18.- Siddiqui AA, Adler DG, Nieto J, Shah J, Binmoeller KF, Kane S, et al. EUSguided drainage of peripancreatic fluid collections and necrosis by using a novel lumen-apposing stent: a large retrospective, multicenter U.S. experience (with videos). Gastrointest Endosc 2016;83:699-707.

19.- Puli SR, Graumlich JF, Pamulaparthy SR, Kalva N. Endoscopic transmural necrosectomy for walled-off pancreatic necrosis: a systematic review and metaanalysis. Can J Gastroenterol Hepatol 2014;28:50-3.

20.- ASGE Standards of Practice Committee, Muthusamy VR, Chandrasekhara V, Acosta RD, Bruining DH, Chathadi KV, et al. The role of endoscopy in the diagnosis and treatment of inflammatory pancreatic fluid collections. Gastrointest Endosc 2016; 83, 481-8.

21.- Navarrete C, Castillo C, Caracci M, Vargas P, Gobelet J, Robles I. Wide percutaneous access to pancreatic necrosis with self-expandable stent: new application (with video). Gastrointest Endosc 2011;73:609-10. 